\title{
Massless collective excitations in frustrated multi-band superconductors
}

\author{
Keita Kobayashi, ${ }^{1}$ Masahiko Machida, ${ }^{1,2}$ Yukihiro Ota, ${ }^{3}$ and Franco Nori ${ }^{3,4}$ \\ ${ }^{1}$ CCSE, Japan Atomic Energy Agency, 5-1-5 Kashiwanoha, Kashiwa, Chiba 277-8587, Japan \\ ${ }^{2}$ Computational Materials Science Research Team, RIKEN AICS, Kobe, Hyogo 650-0047, Japan \\ ${ }^{3}$ CEMS, RIKEN, Saitama 351-0198, JAPAN \\ ${ }^{4}$ Physics Department, University of Michigan, Ann Arbor, Michigan 48109-1040, USA
}

(Dated: May 30, 2017)

\begin{abstract}
We study collective excitations in three- and four-band superconductors with inter-band frustration, which causes neither 0 nor $\pi$ inter-band phases in the superconducting state. Using a low-energy spin-Hamiltonian originating from a multi-band tight-binding model, we find that mass reduction of a Leggett mode occurs in a wide parameter region of this four-band system. As a limitting case, we have a massless Leggett mode. This massless mode is related to the fact that the mean-field energy does not depend on a relative phase of superconducting order parameters. In other words, we find a link of the massless mode with a degeneracy between a time-reversalsymmetry-breaking state (neither 0 nor $\pi$ phases) and a time-reversal-symmetric state (either 0 or $\pi$ phases). Therefore, the mass of collective modes characterizes well the time-reversal symmetry in frustrated multi-band superconductors.
\end{abstract}

PACS numbers: 74.20.-z, 03.75.Kk, 67.10.-j

\section{INTRODUCTION}

Frustration leads to intriguing phenomena in different physical systems 1,2 . Multi-band superconductors/superfluids, such as iron-based materials 3.4 and multi-component ultra-cold atomic gases ${ }^{5-7}$, , can be frustrated many-body systems. Frustration in these systems originates from competitive interaction between different bands/components, not different spatial sites. This curious inter-band frustration allows a time-reversalsymmetry breaking (TRSB) superconducting state ${ }^{\underline{8}-10}$.

Collective excitations characterize well an ordered state in many-body quantum systems. The Leggett mode $\underline{11}-17$ is a characteristic collective excitation in multi-band superconductivity, as well as the NambuGoldstone (NG) mode associated with U(1)-symmetry breaking, and has been studied in multi-band systems such as magnesium diboride $\underline{\underline{18}}$, iron-based materials $\frac{19}{}$, and atomic gases on a honeycomb optical lattice $\underline{20,21}$. The mass of the Leggett mode strongly depends on interband couplings 12 . A recent striking result $\underline{14}$ is that the mass in a three-band system vanishes at the boundary between a time-reversal symmetric (TRS) state and a TRSB state, changing the strength of the inter-band coupling.

In this paper, we study the connection between interband frustration and the mass of collective excitations. To study properties depending on the number of bands, we focus on two cases, as seen in Fig. 1 First, we examine a three-band system as a minimal model for showing the inter-band frustration. Second, we study a four-band system as an example which shows a feature different from the three-band system. Our approach is to make a map from a multi-band tight-binding model to an effective frustrated spin-Hamiltonian. An analogy with a classical spin system is useful for examining multi-band superconductors $\stackrel{10}{\underline{10}}$.
A mean-field theory of the effective spin-Hamiltonian allows us to calculate the superconducting-phase configurations and the collective excitations. Varying the strength of the inter-band couplings, we obtain a phase diagram of the superconducting state. The massless Leggett mode is found at the phase boundaries between the TRSB and TRS states. This result is consistent with the result by $\mathrm{Lin}$ and $\mathrm{Hu}^{14}$. The main result in this paper is that in the four-band system a massless Leggett mode occurs in a parameter region other than the TRSB-TRS phase boundaries. In this region, the mean-field energy for a TRSB state is equal to the one for a TRS state. Therefore, this massless behavior is related to the degenerate superconducting states. Moreover, we characterize this massless mode, from the viewpoint of inter-band symmetry. Thus, we claim that the mass of collective excitations gives an insight into spontaneous-symmetry breaking in the presence of inter-band frustration.

This paper is organized as follows. The effective spin Hamiltonian is derived from a multi-band tight-binding model in Sec. II The formulae for calculating the superconducting order parameter and the collective excitations are derived, with mean-field approximation. In Sec. III, we solve the resultant formulae in a spatially homogeneous case. We show that the massless behaviors of the Leggett mode are associated with energy degeneracy between the TRSB state and the TRS state. Furthermore, we discuss an effect of quantum fluctuations on the massless modes in Sec. IV] Section $\mathrm{V}$ is devoted to the summary.

\section{EFFECTIVE HAMILTONIAN WITH ANTI-FERROMAGNETIC XY INTERACTION}

An effective Hamiltonian is derived from a multi-band tight-binding model, via the second order perturbation. 
This effective model explicitly shows the presence of inter-band frustration, in terms of anti-ferromagnetic $X Y$ interaction. Using the mean-filed approximation, we show the formulae for calculating the superconducting order parameters and the collective excitations in a spatially homogeneous case. We also define a witness for the TRSB state, scalar chiral order parameter. In the subsequent section, we will calculate these equations numerically.

The Hamiltonian is

$$
H=\sum_{\alpha} \sum_{\sigma=\uparrow, \downarrow} h_{\alpha, \sigma}+\sum_{\alpha, \alpha^{\prime}} v_{\alpha, \alpha^{\prime}},
$$

with

$$
\begin{aligned}
& h_{\alpha, \sigma}=-\sum_{<\boldsymbol{i}, \boldsymbol{j}>} t_{\alpha} c_{\alpha, \sigma, i}^{\dagger} c_{\alpha, \sigma, \boldsymbol{j}}-\sum_{\boldsymbol{i}} \mu c_{\alpha, \sigma, \boldsymbol{i}}^{\dagger} c_{\alpha, \sigma, i}, \\
& v_{\alpha, \alpha^{\prime}}=\sum_{\boldsymbol{i}} W_{\alpha \alpha^{\prime}} c_{\alpha, \uparrow, \boldsymbol{i}}^{\dagger} c_{\alpha, \downarrow, i}^{\dagger} c_{\alpha^{\prime}, \downarrow, i} c_{\alpha^{\prime}, \uparrow, \boldsymbol{i}} .
\end{aligned}
$$

The spatial site is $\boldsymbol{i}=\left(i_{x}, i_{y}, i_{z}\right)$. The electron creation (annihilation) operator is $c_{\alpha, \sigma, i}^{\dagger}\left(c_{\alpha, \sigma, i}\right)$ for the $\alpha$ th band on $\boldsymbol{i}$. The hopping matrix element and the chemical potential are, respectively, $t_{\alpha}$ and $\mu$. The intra-band coupling $W_{\alpha \alpha}$ is negative (attractive interaction), while the inter-band coupling $W_{\alpha \alpha^{\prime}}\left(\alpha \neq \alpha^{\prime}\right)$ is positive (repulsive interaction).

Our approach for deriving an effective model from Eq. (11) is the second-order Brillioun-Wigner perturbation. Since strong intra-band coupling produces condensates, our choice of a free Hamiltonian is $H_{0}=\sum_{\alpha} v_{\alpha, \alpha}$. The attractive-repulsive transformation ${ }^{22}$ makes Eq. (1) a half-filled system. This transformation is defined by $c_{\alpha, \uparrow, i}=\bar{c}_{\alpha, \uparrow, \boldsymbol{i}}$ and $c_{\alpha, \downarrow, i}=\exp \left(-i \boldsymbol{q} \cdot \boldsymbol{x}_{\boldsymbol{i}}\right) \bar{c}_{\alpha, \downarrow, \boldsymbol{i}}^{\dagger}$, with a reciprocal vector $\boldsymbol{q}$ satisfying $\exp \left[i \boldsymbol{q} \cdot\left(\boldsymbol{x}_{\boldsymbol{i}+\mathbf{1}_{l}}-\boldsymbol{x}_{\boldsymbol{i}}\right)\right]=-1$, for $l=x, y, z$, where $\boldsymbol{x}_{\boldsymbol{i}}=\sum_{l} i_{l} \boldsymbol{a}_{l}$ and $\mathbf{1}_{l}=\boldsymbol{a}_{l} /\left|\boldsymbol{a}_{l}\right|$. The lattice vector along $l$-axis is $\boldsymbol{a}_{l}$. The ground-state subspace of $H_{0}$ is $\mathcal{H}_{\mathrm{g}}=\otimes_{\alpha, i}\left\{\bar{c}_{\alpha, \uparrow, i}^{\dagger}|\overline{0}\rangle, \bar{c}_{\alpha, \downarrow, i}^{\dagger}|\overline{0}\rangle\right\}$, and the excited-state subspace is $\mathcal{H}_{\mathrm{e}}=\otimes_{\alpha, i}\left\{|\overline{0}\rangle, \bar{c}_{\alpha, \downarrow, i}^{\dagger} \bar{c}_{\alpha, \uparrow, i}^{\dagger}|\overline{0}\rangle\right\}$. The ket vector $|\overline{0}\rangle$ is defined by $\bar{c}_{\alpha, \sigma, i}|\overline{0}\rangle=0$. The effective Hamiltonian is $H_{\text {eff }}=P V P-(P V Q) H_{0}^{-1}(Q V P)$, with $V=H-H_{0}$. The projector onto $\mathcal{H}_{\mathrm{g}}\left(\mathcal{H}_{\mathrm{e}}\right)$ is $P(Q)$.

Let us write $H_{\text {eff }}$ in terms of the pseudo-spin 1/2 operators defined by $\bar{S}_{\alpha, i}^{(+)}=\bar{c}_{\alpha, \uparrow, i}^{\dagger} \bar{c}_{\alpha, \downarrow, i}, \bar{S}_{\alpha, i}^{(-)}=\left[\bar{S}_{\alpha, i}^{(+)}\right]^{\dagger}$, and $\bar{S}_{\alpha, \boldsymbol{i}}^{(z)}=\left(\bar{c}_{\alpha, \uparrow, i}^{\dagger} \bar{c}_{\alpha, \uparrow, i}-\bar{c}_{\alpha, \downarrow, i}^{\dagger} \bar{c}_{\alpha, \downarrow, i}\right) / 2$. These operators represent a fermion-pair particle. The 2 nd perturbation term leads to the Heisenberg Hamiltonian with exchange interaction $J_{\alpha}=2 t_{\alpha}^{2} /\left|W_{\alpha \alpha}\right|$. The contribution from $W_{\alpha \alpha^{\prime}}\left(\alpha \neq \alpha^{\prime}\right)$ appears as the 1st perturbation term since $P v_{\alpha, \alpha^{\prime}} Q=0$ for $\alpha \neq \alpha^{\prime}$. Thus,

$$
\begin{aligned}
H_{\mathrm{eff}}= & \sum_{\alpha} \sum_{<\boldsymbol{i}, \boldsymbol{j}>} J_{\alpha}\left[\bar{S}_{\alpha, \boldsymbol{i}}^{(z)} \bar{S}_{\alpha, \boldsymbol{j}}^{(z)}+\bar{S}_{\alpha, \boldsymbol{i}}^{(+)} \bar{S}_{\alpha, \boldsymbol{j}}^{(-)}\right] \\
& +\sum_{\alpha \neq \alpha^{\prime}} \sum_{\boldsymbol{i}} W_{\alpha \alpha^{\prime}} \bar{S}_{\alpha, \boldsymbol{i}}^{(+)} \bar{S}_{\alpha^{\prime}, \boldsymbol{i}}^{(-)}-\sum_{\alpha, \boldsymbol{i}} 2 \bar{\mu}_{\alpha} \bar{S}_{\alpha, \boldsymbol{i}}^{(z)},
\end{aligned}
$$

(a)

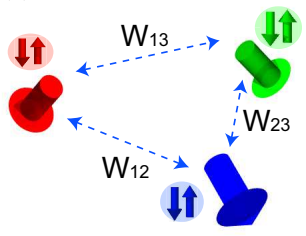

(b)

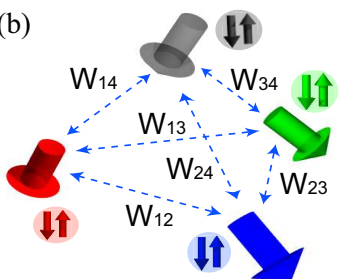

FIG. 1: (Color online) Schematic diagrams of inter-band configurations in (a) three- and (b) four-band systems. Each large arrow indicates the orientation of a pseudo-spin formed by a fermion-pair particle on a band. Since inter-band couplings $W_{\alpha \alpha^{\prime}}$ are repulsive, an anti-ferromagnetic interaction occurs between the pseudo-spins. See Eq. (4).

with $\bar{\mu}_{\alpha}=\mu+\left|W_{\alpha \alpha}\right| / 2$. The inter-band interaction is regarded as an anti-ferromagnetic $X Y$-interaction.

We examine Eq. (44), using the mean-field approach with spatial uniformity. Let us rewrite the pseudo-spin- $1 / 2$ operators, in terms of $b_{\alpha, i}$, such that $\bar{S}_{\alpha, \boldsymbol{i}}^{(+)}=\exp \left(-\mathrm{i} \boldsymbol{q} \cdot \boldsymbol{x}_{\boldsymbol{i}}\right) b_{\alpha, \boldsymbol{i}}$ and $\quad \bar{S}_{\alpha, \boldsymbol{i}}^{(z)}=(1 / 2)-b_{\alpha, \boldsymbol{i}}^{\dagger} b_{\alpha, \boldsymbol{i}}$. We find that $\left[b_{\alpha, \boldsymbol{i}}, b_{\alpha^{\prime}, \boldsymbol{i}^{\prime}}^{\dagger}\right]=\left(1-b_{\alpha, \boldsymbol{i}}^{\dagger} b_{\alpha, \boldsymbol{i}}\right) \delta_{\boldsymbol{i} \boldsymbol{i}^{\prime}} \delta_{\alpha \alpha^{\prime}}$ and $b_{\alpha, i}^{2}=0 . \quad$ In the dilute limit $\left\langle b_{\alpha, i}^{\dagger} b_{\alpha, i}\right\rangle \ll 1, b_{\alpha, i}$ can be regarded as a standard bosonic operator. Using $\left\langle b_{\alpha, i}\right\rangle=\Delta_{\alpha, i}$, we obtain the mean-field energy $E_{\mathrm{c}}$ as a function of $\Delta_{\alpha, i}$. For the uniform order parameters $\left(\Delta_{\alpha, i}=\Delta_{\alpha}\right), \Delta_{\alpha}$ is determined by $\left(\partial E_{\mathrm{c}} / \partial \Delta_{\alpha}^{*}\right)=0$, namely,

$$
-2 J_{\alpha} D\left(1-2\left|\Delta_{\alpha}\right|^{2}\right) \Delta_{\alpha}+\sum_{\alpha^{\prime} \neq \alpha} W_{\alpha \alpha^{\prime}} \Delta_{\alpha^{\prime}}-\nu_{\alpha} \Delta_{\alpha}=0,(5)
$$

where $\nu_{\alpha}=2 D J_{\alpha}-2 \bar{\mu}_{\alpha}$ and $D$ is the dimension of the system. The collective excitations for momentum $\boldsymbol{k}$ are calculated, combining the resultant gaps with the Bogolubov de-Gennes equation

$$
T_{k} \boldsymbol{Y}_{\boldsymbol{k}}=\omega_{\boldsymbol{k}} \boldsymbol{Y}_{\boldsymbol{k}}
$$

with $T_{\boldsymbol{k}}=\tau_{z} \otimes \mathcal{L}+\tau_{x} \otimes i \operatorname{Im} \mathcal{M}+\tau_{y} \otimes i \operatorname{Re} \mathcal{M} . \quad \boldsymbol{Y}_{\boldsymbol{k}}$ is a $2 N$-complex vector, where $N$ is the number of bands. The $2 \times 2$ Pauli matrices $\left(\tau_{x}, \tau_{y}, \tau_{z}\right)$ represent the so-called particle-hole symmetry of the Bogoliubovde Gennes equation. The $N \times N$ matrices $\mathcal{L}$ and $\mathcal{M}$ are defined as,

$$
\begin{aligned}
\mathcal{L}_{\alpha \alpha^{\prime}}= & -2 \delta_{\alpha, \alpha^{\prime}} \sum_{l}\left[\varepsilon_{\alpha, k_{l}}-2\left(\varepsilon_{\alpha, k_{l}}-\varepsilon_{\alpha, 0}\right)\left|\Delta_{\alpha}\right|^{2}\right] \\
& -\delta_{\alpha, \alpha^{\prime}} \nu_{\alpha}+\left(1-\delta_{\alpha, \alpha^{\prime}}\right) W_{\alpha \alpha^{\prime}} \\
\mathcal{M}_{\alpha \alpha^{\prime}}= & \delta_{\alpha, \alpha^{\prime}} \sum_{l} 4 \varepsilon_{\alpha, k_{l}} \Delta_{\alpha}^{2} .
\end{aligned}
$$

The coefficient $\varepsilon_{\alpha, k_{l}}$ is the Fourier-transformed hopping matrix element, $\varepsilon_{\alpha, k_{l}}=J_{\alpha} \cos \left(k_{l} a_{l}\right)$, with lattice constant $a_{l}\left(=\left|\boldsymbol{a}_{l}\right|\right)$.

The superconducting states are classified by the scalar chiral order parameter $\underline{23,24}$

$$
\chi=\sum_{\alpha_{1}<\alpha_{2}<\alpha_{3}}\left|\left\langle\overline{\boldsymbol{S}}_{\alpha_{1}} \cdot\left(\overline{\boldsymbol{S}}_{\alpha_{2}} \times \overline{\boldsymbol{S}}_{\alpha_{3}}\right)\right\rangle\right| .
$$



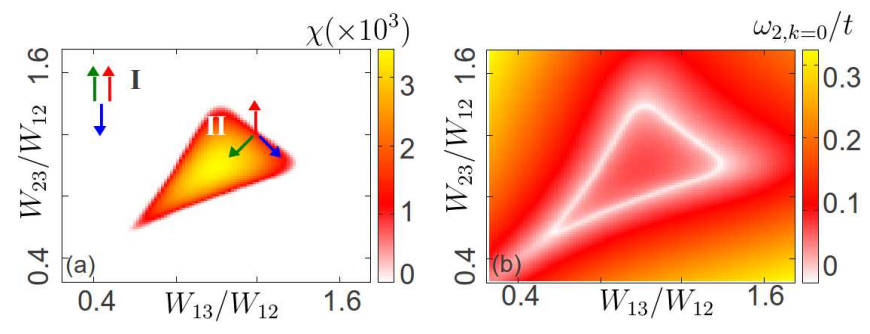

FIG. 2: (Color online) Density profiles of (a) a scalar chiral order parameter and (b) Leggett-mode mass, in a three-band superconductor, varying inter-band couplings $W_{13} / W_{12}$ and $W_{23} / W_{12}$. In (a), the arrows show typical superconductingphase configurations.

Under the mean-field approximation and the dilute limit, the components of the pseudo-spin $1 / 2$ vector $\overline{\boldsymbol{S}}_{\alpha}$ are $\overline{\boldsymbol{S}}_{\alpha} \simeq\left\langle\overline{\boldsymbol{S}}_{\alpha}\right\rangle={ }^{\mathrm{t}}\left(\Delta_{\alpha}^{\mathrm{R}}, \Delta_{\alpha}^{\mathrm{I}}, 1 / 2-\left|\Delta_{\alpha}\right|^{2}\right) \simeq{ }^{\mathrm{t}}\left(\Delta_{\alpha}^{\mathrm{R}}, \Delta_{\alpha}^{\mathrm{I}}, 1 / 2\right)$, where $\Delta_{\alpha}^{\mathrm{R}}$ and $\Delta_{\alpha}^{\mathrm{I}}$ are, respectively, the real and the imaginary parts of $\Delta_{\alpha}$. The inter-band phases (e.g., $\left.\Delta_{1}^{\mathrm{R}} \Delta_{2}^{\mathrm{I}}-\Delta_{1}^{\mathrm{I}} \Delta_{2}^{\mathrm{R}}\right)$ are important for determining the TRSB state. We sum up such quantities over all the band indices in Eq. (9). We note that $\chi=0$ when $\Delta_{\alpha}^{\mathrm{I}}=0$ for all $\alpha$.

\section{MASS REDUCTION OF A LEGGETT MODE BY INTER-BAND FRUSTRATION}

We calculate the scalar chiral order parameter on a $2 \mathrm{D}$ square lattice $\left(D=2\right.$ and $\left.a_{l}=a\right)$, numerically solving Eq. (5), according to the imaginary-time evolution method 25,26 . We also evaluate the collective modes by direct diagonalization of Eq. (6). Here, we consider in a highly symmetric case $t_{\alpha}=t$ for simplicity and focus on a strong intra-band interaction case, $W_{\alpha \alpha} / t=-6$, to ensure the validity of Eq. (4). Throughout this paper, we set $J_{\alpha} / t=J / t=1 / 3$. The condensate particle-density is also fixed as $n_{\mathrm{c}}=\sum_{\alpha}\left|\Delta_{\alpha}\right|^{2}=0.1$. The number of the collective modes depends on $N$. We will denote the NG mode as $\omega_{1, \boldsymbol{k}}$. The others correspond to the Leggett modes.

First, we show the results for the three-band case. Figure 2(a) shows the presence of different parameter regions. In region I the TRS states occur $(\chi=0)$, whereas in region II the TRSB states occur $(\chi \neq 0)$. In region I a sign change (anti-parallel arrangement of pseudo-spins) occurs between the gaps. In region II a typical phaseconfiguration is that each relative superconducting phase is $2 \pi / 3$. In other words, each pseudo-spin directs from the center to the vertex of an equilateral triangle. Figure 2 (b) shows that the mass of the Leggett mode $\left(\omega_{2, \boldsymbol{k}=0}\right)$ vanishes at the TRSB-TRS phase boundaries. These results are consistent with the results of a weak-coupling model $\stackrel{14}{ }$. The phase transition between the TRS and the TRSB state is the 2nd-order one, as shown by Lin and $\mathrm{Hu}^{14}$. The fluctuation developed at the critical point may lead to this massless behavior.

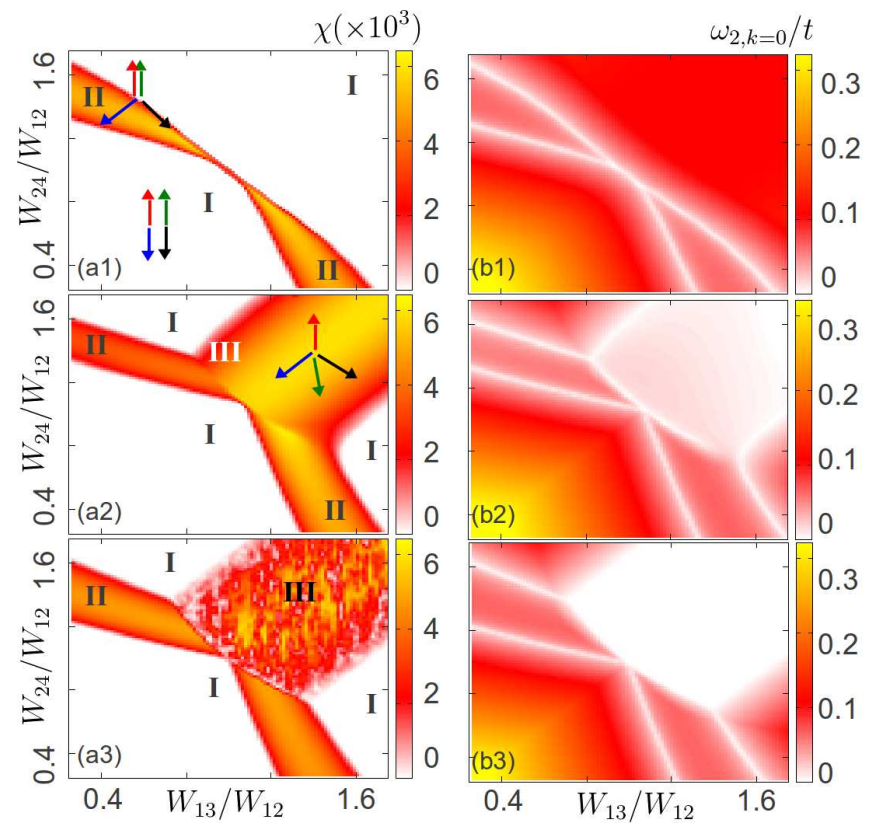

FIG. 3: (Color online) Density profiles of (a1,a2,a3) a scalar chiral order paramter and (b1,b2,b3) Leggett-mode mass, in a four-band superconductor, varying inter-band couplings $W_{13}$ and $W_{24}$. The other inter-band couplings are fixed as $W_{12}=$ $W_{34}=0.23, W_{23}=W_{14}=0.2$ in (a1,b1), $W_{12}=W_{23}=0.23$, $W_{34}=W_{14}=0.2$ in $(\mathrm{a} 2, \mathrm{~b} 2)$, and $W_{12}=W_{23}=W_{34}=$ $W_{14}=0.2$ in $(\mathrm{a} 3, \mathrm{~b} 3)$. The arrows in $(\mathrm{a} 1, \mathrm{a} 2)$ show typical superconducting-phase configurations like Fig.2

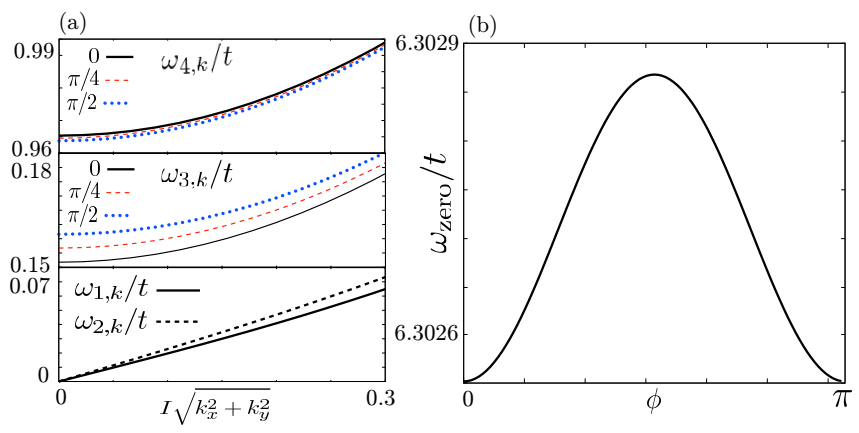

FIG. 4: (Color online) (a) Dispersion relations for collective excitations, with relative phases $\phi=0, \pi / 4, \pi / 2 . \omega_{1, k}$ and $\omega_{2, k}$ are independent of $\phi$. (b) Zero-point energy of the collective excitations, varying $\phi$. In both figures, inter-band couplings are set as $W_{13} / W_{12}=1.2, W_{24} / W_{12}=1.3$, and $W_{12}=W_{23}=W_{34}=W_{14}=0.2$.

Now, let us show the four-band case. We change $W_{13}$ and $W_{24}$, with fixed $W_{12}, W_{23}, W_{34}$ and $W_{14}$. From the viewpoint of Fig. 1, the length of four sides in a tetrahedron is fixed. First, we show similar features to the three-band case. Figure 3(a1) shows the results for $W_{12}=W_{34}=0.23$ and $W_{23}=W_{14}=0.2$. The TRSB state appears in region $\mathbb{I}$, whereas the TRS states occur in the other regions. The phase configuration in region II is similar to the three-band case, 
although two of the pseudo-spins are aligned (0-phase shift). We also find that the mass of the Leggett mode vanishes at the TRSB-TRS phase boundaries, as seen in Fig. 3(b1). Changing the condition for the fixed interband couplings, different features appear. Let us consider the case of $W_{12}=W_{23}=0.23$ and $W_{34}=W_{14}=0.2$. Figure 3(a2) shows the presence of a curious area (region III), where the time-reversal symmetry is fully broken. In other words, every relative phase is neither 0 nor $\pi$. Figure 3(b2) shows that the mass of the Leggett mode is close to zero inside this region. We can also find that $\omega_{2, \boldsymbol{k}=0}$ and $\omega_{3, \boldsymbol{k}=0}$ become zero near the phase boundaries between II and III (no figure shown for $\omega_{3, \boldsymbol{k}}$ ). A more exotic feature appears in the identical inter-band interaction $W_{12}=W_{23}=W_{34}=W_{14}=0.2$. Figure 33 (a3) shows that $\chi$ randomly changes in region $\mathrm{II}^{26} . \omega_{2, \boldsymbol{k}}$ is massless in this wide area, not restricted near the phase boundaries.

We examine region III in Fig. [3(a3) in detail. Since $W_{13}>W_{12}$, a $\pi$-shift may occur between $\Delta_{1}$ and $\Delta_{3}$. Similarly, the condition $W_{24}>W_{12}$ means $\Delta_{4}=e^{ \pm i \pi} \Delta_{2}$. Moreover, since $W_{12}=W_{34}$, the relative phase between $\Delta_{1}$ and $\Delta_{2}$ should be equal to the one between $\Delta_{3}$ and $\Delta_{4}$. Therefore, we construct a solution of Eq. (5) in region III, with ansatz

$$
\boldsymbol{\Delta}=\left(\left|\Delta_{+}\right|, e^{i \phi}\left|\Delta_{-}\right|, e^{i \pi}\left|\Delta_{+}\right|, e^{i(\phi+\pi)}\left|\Delta_{-}\right|\right) .
$$

The global phase is fixed so that $\Delta_{1}$ is real. Substituting this expression into Eq. (5), we find that $\left|\Delta_{ \pm}\right|=(1 / 2) \sqrt{n_{\mathrm{c}} \pm\left(W_{13}-W_{24}\right) / 2 J D}$, but the relative phase is not fixed. This result indicates that the meanfield energy for Eq. (10) is independent of the continuous parameter $\phi$, and a degeneracy exists between the TRSB and the TRS states. Thus, the massless behavior in region III is related to a degeneracy. The occurrence of such an exotic massless mode and a degeneracy between ground states were pointed out by several authors ${ }^{27-30}$.

A symmetry analysis of Eq. (6) leads to insights into the massless Leggett mode. The identical inter-band couplings $\left(W_{12}=W_{23}=W_{34}=W_{14}\right)$ and the order parameters (10) indicate the presence of a symmetric property in Eq. (6). We find that $\mathcal{L}=\mathbb{1} \otimes \mathcal{L}_{0}+\eta_{x} \otimes \mathcal{L}_{x}$ and $\mathcal{M}=\mathbb{1} \otimes \mathcal{M}_{0}$, with the $x$-component of the $2 \times 2$ Pauli matrices, $\eta_{x}$ and complex $2 \times 2$ matrices $\mathcal{L}_{0}, \mathcal{L}_{x}$, and $\mathcal{M}_{0}$. Hence, $\eta_{x}$ commutes with $T_{\boldsymbol{k}}$. We mention that $\eta_{x}$ corresponds to swap between the upper 2-band and the lower 2-band blocks. After a permutation, we rewrite $T_{\boldsymbol{k}}$ as

$$
T_{k}=\frac{\mathbb{1}+\eta_{x}}{2} \otimes T_{+, k}+\frac{\mathbb{1}-\eta_{x}}{2} \otimes T_{-, k},
$$

with $T_{ \pm, \boldsymbol{k}}=\operatorname{tr}_{\eta}\left[T_{\boldsymbol{k}}\left(\mathbb{1} \pm \eta_{x}\right)\right] / 2$. The symbol $\operatorname{tr}_{\eta}$ means the trace over $\eta$-basis. The characteristic polynomial of $T_{-, \boldsymbol{k}}$ is written by $f(z)=\sum_{n=0}^{4} c_{n}(\boldsymbol{k}) z^{4-n}$, with $c_{0}=1$. Since we can find that the coefficients for $n=1,2,3$ are zero when $\boldsymbol{k}=0, T_{\boldsymbol{k}}$ has two zero modes, one of which is the NG mode, while the other of which is the massless Leggett mode. Thus, the present massless Leggett mode belongs to the same subspace as the NG mode, and is regarded as a quasi-NG mode.

\section{DISCUSSION}

We refer to an effect of quantum fluctuations on the ground-state degeneracy. The simplest approach to take such corrections is to add the zero-point energy of the collective excitations to the mean-field energy. The correction can be written as $\omega_{\text {zero }}=\sum_{\alpha} \sum_{k} \omega_{\alpha, \boldsymbol{k}} / I$, with the total number $I$ of the spatial sites. Let us examine this correction in region III of Fig. 33(a3). Figure 4(a) shows that $\omega_{3, \boldsymbol{k}}$ and $\omega_{4, \boldsymbol{k}}$ depend on $\phi$, whereas the others not so. Figure $4(\mathrm{~b})$ shows $\omega_{\text {zero }}$ has minimum values at either 0 or $\pi$. In other words, the massive collective modes in region III make a selection of a true groundstate. The TRS state is preferable in region III, owing to $\omega_{\text {zero. }}$.

The above consideration indicates that our quasi-NG mode may obtain some mass originating from quantum fluctuations. This point is also discussed in a different system, spinor Bose-Einstein condensate 28 . Nevertheless, the mass of the Leggett mode is a good indicator of inter-band frustration. Indeed, our calculations show that the mass of the Leggett mode drastically reduces (almost zero) when strong competition between the inter-band couplings, even though the ground-state degeneracy is absent. See region III of Fig. 3(b2), for example. In this region, only the TRSB state occurs; it means that strong inter-band frustration appears. Thus, although the Leggett mode does not become a compelte massless mode in the presence of quantum fluctuations, one may observe a significant mass reducing behavior of a Leggett mode. When the long-range Coulomb interaction exists, the situation becomes much clearer. Typically, the NG mode obtains the mass via the AndersonHiggs mechanism; the massive plasma excitations may appear. However, since the Leggett modes are related to neutral superfluid-phase fluctuations $\frac{12}{2}$, one may observe low-energy excitations related to the Leggett mode with tiny mass, whenever strong inter-band frustration exists. Therefore, we expect that the mass reducing behavior of the Leggett mode predicted by the present mean-field analysis is robust against quantum fluctuations and the gauge filed. A more systematic study about different fluctuations is an interesting future work.

\section{SUMMARY}

We have examined the collective excitations in threeand four-band superconductors. Using an effective spin Hamiltonian, we showed that inter-band frustration induces two kinds of massless Leggett modes, and clarified their physical origin. The mass of a collective mode characterizes well the time-reversal symmetry of frustrated multi-band superconductors. 


\section{Acknowledgments}

We thank M. Okumura and H. Nakamura for useful discussions. This work was partially supported by MEXT Strategic Programs for Innovative Research, and the Computational Materials Science Initiative, Japan. We are indebted to T. Toyama for his support. Y.O. is partially supported by the Special Postdoctoral Researchers Program, RIKEN. F.N. acknowledges partial support from the ARO, RIKEN iTHES project, JSPSRFBR Contract No. 12-02-92100, Grant-in-Aid for Scientific Research (S), MEXT Kakenhi on Quantum Cybernetics, and Funding Program for Innovative R\&D on S\&T.
1 Frustrated Spin Systems, edited by H. T. Diep (World Scientific, Singapore, 2004).

2 M. S. Li, Phys. Rep. 376133 (2003).

3 K. Ishida, Y. Nakai, and H. Hosono, J. Phys. Soc. Jpn. 78, 062001 (2009).

4 J. Paglione and R. L. Greene, Nature Phys. 6, 645 (2010).

5 T. Müller, S. Fölling, A. Widera, and I. Bloch, Phys. Rev. Lett. 99, 200405 (2007).

6 A. V. Gorshkov, M. Hermele, V. Gurarie, C. Xu, P. S. Julienne, J. Ye, P. Zoller, E. Demler, M. D. Lukin, and A. M. Rey, Nature Phys. 6, 289 (2010).

7 G. Wirth, M. Ölschläger, and A. Hemmerich, Nature Phys. 7, 147 (2011).

8 V. Stanev and Z. Tesanovic, Phys. Rev. B. 81, 134522 (2010).

9 Y. Tanaka and T. Yanagisawa, J. Phys. Soc. Jpn. 78, 062001 (2009).

10 R. G. Dias and A. M. Marques, Supercond. Sci. Technol, 24, 085009 (2011).

11 A. J. Leggett, Prog. Theor. Phys. 36, 901 (1966).

12 Y. Ota, M. Machida, T. Koyama, and H. Aoki, Phys. Rev. B. 83, 060507(R) (2011).

13 J. Carlström, J. Garaud, and E. Babaev, Phys. Rev. B 84, 134518 (2011).

14 S.-Z. Lin and X. Hu, Phys. Rev. Lett. 108, 177005 (2012).

15 V. Stanev, Phys. Rev. B 85, 174520 (2012).

16 T. Yanagisawa and I. Hase, J. Phys. Soc. Jpn. 82, 124704 (2013).

17 D. Weston and E. Babaev, arXiv: 1306.3179 (unpublished).

18 G. Blumberg, A. Mialitsin, B. S. Dennis, M. V. Klein, N. D. Zhigadlo, and J. Karpinski, Phys. Rev. Lett. 99, 227002
(2007).

19 F. J. Burnell, J. Hu, M. M. Parish, and B. A. Bernevig, Phys. Rev. B 82, 144506 (2010).

20 E. Zhao and A. Paramekanti, Phys. Rev. Lett. 97, 230404 (2006).

21 S. Tsuchiya, R. Ganesh, and A. Paramekanti, Phys. Rev. A 86, 033604 (2012).

22 R. Micnas, J. Ranninger, and S. Robaszkiewicz, Rev. Mod. Phys. 62, 113 (1990).

${ }^{23}$ X. G. Wen, F. Wilczek, and A. Zee, Phys. Rev. B 39, 11413 (1989).

24 This definition is different from the conventional one ${ }^{23}$. We take the absolute value of $\left\langle\overline{\boldsymbol{S}}_{\alpha_{1}} \cdot\left(\overline{\boldsymbol{S}}_{\alpha_{2}} \times \overline{\boldsymbol{S}}_{\alpha_{3}}\right)\right\rangle$.

25 A. Minguzzi, S. Succi, F. Toschi, M. P. Tosi, and P. Vignolo, Phys. Rep. 395, 223 (2004).

${ }^{26}$ We randomly change the initial order parameter, solving Eq. (5) in the imaginary-time evolution method. Whenever there is no degeneracy between the TRSB state and the TRS state, the unique solution is determined for every initial choice. However, when the degeneracy exits, the resultant scalar chiral order parameter $\chi$ depends on the choice, as a result $\chi$ randomly changes, as seen in region III of Fig. 3(a3).

27 J. L. Song, G. W. Semenoff, and F. Zhou, Phys. Rev. Lett. 98, 160408 (2007).

28 S. Uchino, M. Kobayashi, M. Nitta, and M. Ueda, Phys. Rev. Lett. 106, 230406 (2010).

29 Z. Cai, Y. Wang, and C. Wu, Phys. Rev. B. 86, 060517(R) (2012).

30 O. A. Castro-Alvaredo and B. Doyon, Phys. Rev. Lett. 108, 120401 (2012). 